\title{
Differential integration efforts of mandatory and optional sentence constituents
}

\author{
ANAT PRIOR $^{\mathrm{a}}$ AND SHLOMO BENTIN ${ }^{\mathrm{b}}$ \\ ${ }^{a}$ Department of Psychology, Carnegie Mellon University, Pittsburgh, Pennsylvania, USA \\ ${ }^{\mathrm{b}}$ Department of Psychology and The Interdisciplinary Center for Neural Computation, Hebrew University of Jerusalem, Jerusalem, Israel
}

\begin{abstract}
We investigated the online sensitivity of the semantic integration system to the different roles played by sentence constituents that are necessary (verbs and nouns) or optional (adjectives) for argument completion. We compared the effect of semantic incongruities introduced in both types of words on the N400 ERP component. Participants read sentences for meaning, half of which were rendered anomalous by an incongruent verb, noun, or an early/late adjective. Incongruent adjectives led to smaller N400 effects than did incongruent nouns and verbs, and the congruity effect for sentence-final adjectives was not significant. All incongruities are therefore not created equal: Incongruent optional sentence constituents create less of an integrative burden than incongruent mandatory sentence constituents, suggesting that online sentence integration processes are sensitive to the distinct roles played by different words in shaping sentence meaning.
\end{abstract}

Descriptors: N400, ERP, Semantic integration, Sentence comprehension, Word class

The current study was designed to compare the relative importance of verbs, nouns, and adjectives in shaping the meaning of simple transitive sentences. We gauged the online sensitivity of the semantic integration system to the different roles played in the construction of sentence meaning by sentence constituents that are mandatory for argument completion (verbs and nouns) or are optional (adjectives).

Semantically and syntactically ambiguous constructions of various complexities have been investigated extensively with the goal of revealing default processing strategies. Factors such as degree of complexity and ambiguity of syntactic construction, referential processing, and the semantic syntactic interface of ambiguity resolution have all been demonstrated to have significant effects on integration and comprehension (Grodner, Gibson, \& Tunstall, 2002; Kaan \& Swaab, 2003; Kuperberg, Sitnikova, Caplan, \& Holcomb, 2003; MacDonald, Pearlmutter, \& Seidenberg, 1994; Trueswell, Tanenhaus, \& Garnsey, 1994; Warren \& Gibson, 2002). In continuation of this research, the current study examined simple active transitive constructions to

This research was funded by the German-Israeli Science Foundation grant \#567, by NICHD grant 01994 to S. Bentin through Haskins Laboratories, New Haven, Connecticut, and by a doctoral research stipend to A. Prior from the Israeli Foundation Trustees. We thank Ayelet Landau for helpful comments and discussions and Osnat Mussel and Gadi Leshman for skillful research assistance. We also thank Dr. Patricia Carpenter and two anonymous reviewers for many valuable suggestions.

Address reprint requests to: Anat Prior, Department of Psycholgy, Carnegie Mellon University, 5000 Forbes Ave., Pittsburgh, PA 15213, USA. E-mail: aprior@andrew.cmu.edu. investigate the different roles played by mandatory and optional sentence constituents in determining sentence meaning.

Linguistic theories have assigned verbs a pivotal role in sentence interpretation (Chomsky, 1981; Dowty, 1991; Levin, 1993). Psycholinguistic research has empirically supported this position showing the importance of verbs in determining thematic roles (Altmann \& Kamide, 1999; Ferretti, McRae, \& Hatherell, 2001; Fisher, Gleitman, \& Gleitman, 1991; Myers \& Blumstein, 2005). Nouns are similarly important in realizing these arguments and specifying the entities involved (MacDonald et al., 1994; Trueswell et al., 1994). Consequently, a semantically incongruent verb or noun should disrupt the main proposition or argument relation described in the sentence. In contrast, based on various accounts of sentence comprehension processes (e.g., Carpenter, Miyake, \& Just, 1995; Clifton \& Duffy, 2001; Townsend \& Bever, 2001), we hypothesized that semantically incongruent adjectives would interrupt sentence integration to a lesser degree than would semantically incongruent verbs and nouns. This is because in simple transitive sentences, semantically incongruent adjectives induce only a local anomaly, limited to a single thematic role within the sentence, be it the agent or the patient. We addressed these hypotheses by comparing the effect of semantic incongruities introduced in different sentence constituents on the N400 ERP measure of semantic integration.

Event-related brain potentials (ERPs) have been used extensively in psycholinguistic research (e.g., Bentin, MouchetantRostaing, Giard, Echallier, \& Pernier, 1999; for reviews see Brown \& Hagoort, 1999; Friederici, 2004; Hinojosa, MartinLoeches, \& Rubia, 2001; Kutas, Federmeier, Coulson, King, \& Munte, 2000; Kutas \& Van Petten, 1994; Osterhout \& Holcomb, 
1995). Specifically, a large proportion of ERP studies of language have examined sentence or discourse processing (for recent reviews, see Osterhout, Allen, \& McLaughlin, 2002; van Berkum, Zwitserlood, Hagoort, \& Brown, 2003) with a focus on sentential semantic integration (Kutas \& King, 1996). Many of these studies examined the N400 component, whose association with semantic processing was first demonstrated by Kutas and Hillyard (1980; for recent findings, see, e.g., van Berkum et al., 2003). The amplitude of the N400 is modulated by the semantic congruity of a word with preceding context, with a greater negativity exhibited for stimuli that are difficult to integrate in the semantic structure set by the sentence (Frisch, Hahne, \& Friederici, 2004; van Berkum, Hagoort, \& Brown, 1999). In addition, the N400 is sensitive to how much an upcoming word in a sentence is expected. The amplitude of the N400 has a strong negative correlation with the cloze probability of semantically acceptable sentence-embedded words (Kutas \& Hillyard, 1984; Kutas, Lindamood \& Hillyard, 1984; replicated recently by DeLong, Urbach, \& Kutas, 2005). The more predictable a word is, as indexed by off-line sentence completion measures, the smaller the N400 amplitude. However, cloze probability and expectancy are not the only factors affecting the N400. Indeed, N400 modulations have also been demonstrated for semantically incongruent words that were equally unexpected (cloze probabilit $y=0$ ). For example, the amplitude of the N400 to incongruent items that are semantically related to the expected word is smaller than to other incongruent items (Kutas \& Hillyard, 1980). Hence, current models assume that the modulation of the N400 by sentence-embedded words indexes the ease with which the word can be integrated online into sentential semantic context (van Berkum et al., 1999).

In the current work, we further examined the sensitivity of the N400 to different degrees of semantic incongruity induced by words that had a cloze probability of zero, that is, were deemed incongruent with preceding context. The critical manipulation was the degree of anomaly induced by the incongruity, which was dictated by the syntactic/thematic category of the incongruous word. Incongruous nouns and verbs should have global effects on meaning computation, and therefore might require a larger integration effort evident in larger N400. Incongruous adjectives, on the other hand, should prove to be less detrimental to the integration process, and therefore trigger a smaller effect on the N400 (for related findings with single words, see Titone \& Salisbury, 2004).

The question of whether the N400 component is sensitive to word class per se has received a fair degree of attention in the literature. Most studies have contrasted open- and closed-class words, focusing both on an early anterior negativity, labeled the $\mathrm{N} 280$, and on differences in the N400. Some have claimed the N280 to be a distinct marker of closed-class words (e.g., Neville, Mills, \& Lawson, 1992) whereas others disputed these results, finding no evidence for this component in response to closedclass words (Osterhout et al., 2002) or suggesting that the N280 is evident for both open- and closed-class words (e.g., Brown, Hagoort, \& ter Keurs, 1999). There is generally better agreement concerning the N400, with open-class words usually eliciting more negative N400s than closed-class words (Brown et al., 1999; McCarthy, Nobre, Bentin, \& Spencer, 1995), though some authors have claimed that these findings reflect differences in word frequency (Munte et al., 2001) or length (Osterhout et al., 2002) between open- and closed-class words, rather than manifestations of distinct brain processing based on word class.
Recently, differences were also found among the N400 evoked by different open-class words (see Brown et al., 1999, for a comparison of contextually- congruent nouns, verbs, and adjectives), specifically when nouns and verbs are compared (Federmeier, Segal, Lombrozo, \& Kutas, 2000; Pulvermuller, Lutzenberger, \& Preissl, 1999). However, in the present experiment we did not emphasize the overall differences in the ERPs to different categories of open-class words. Rather, we used modulations of the N400 component as a dependent measure indexing the degree of semantic incongruity created by violating selectional restrictions of different sentence constituents, and investigated the consequences of these incongruities for sentence integration. Federmeier et al. (2000) demonstrated larger N400 effects for incongruities at the point of an expected verb as compared to incongruities at the point of an expected noun. However, in that study the incongruent item was either a pseudoword or a word from the opposite word class (i.e., a noun instead of an expected verb and vice versa). Thus, the study did not tap the issue of semantic integration in isolation, but examined it conjointly with syntactic integration difficulties. Further, adjectives were not used as target items in that study, which does not allow for a comparison between the effect of incongruities in mandatory and optional sentence constituents.

In the Hebrew sentences used in this study, the verb and the object noun were mandatory sentential constituents necessary to complete the basic argument structure, whereas the adjectives were optional sentence constituents, as is normally the case. In English, adjectives usually precede the noun they are modifying (as is the case in German and in Dutch) and thus it is more difficult to generate experimental materials in which the meaning violation is apparent upon processing of the adjective itself (indeed, we have not been able to find any direct references to studies using such materials). Note that this is opposed to many studies that did employ adjective-noun agreement violations (cf. Hagoort, 2003), where the reader realizes that there is an incongruity only once she or he is exposed to the modified noun. In Hebrew, however, adjectives normally follow the noun they are modifying, making it very straightforward to generate materials in which the adjective is the word causing the incongruity with a noun that has already been processed.

The sentences constructed for this study were initially presented to participants for an off-line plausibility rating, to obtain a preliminary measure of the degree of anomaly created by the noun, verb, and adjective incongruities. To anticipate, the rating procedure confirmed our initial hypothesis regarding the differential effect of the three incongruity types on sentence coherence. However, the rating was only considered a first approximation because off-line judgments can often be influenced by post-sentence analysis processes, which do not necessarily reflect differences in the online processing and comprehension of each incoming word of the sentence. Therefore we went on to record other participants' brain activity while reading these sentences for meaning.

\section{Method \\ Participants}

Twenty native Hebrew speakers, undergraduates at the Hebrew University, with normal or corrected-to-normal vision, participated in the experiment for course credit or payment. 


\section{Task and Design}

Syntactically intact sentences were presented one word at a time at the center of a computer screen. Half of the sentences in each experimental list were semantically plausible and half included a semantic incongruity involving a noun, a verb, or an adjective (see details below). Participants were instructed to read the sentences and to respond to occasional questions regarding their meaning. Such questions appeared randomly in $12.5 \%$ of the trials (eight questions in each block of 48 trials). The questions tested sentence comprehension; they focused on all sentence constituents equally and were as likely to appear following coherent and semantically anomalous sentences.

Two experimental lists were constructed, each including 288 sentences. In each list, one half of the target stimuli from each word class appeared in coherent sentences and the other half appeared in semantically incongruent sentences. Half of the participants were tested with each list. Therefore, across participants, each word occurred equally in both conditions, though no participant saw any of the target items more than once. More importantly, across participants the congruent and incongruent conditions in each word class were comprised of the very same items, which served as their own control.

\section{Material}

The sentences used were simple active transitive subject-verbobject (SVO) sentences in Hebrew, with adjectives modifying both the subject and the object nouns. The critical target words were 72 adjectives modifying the subject, 72 transitive verbs, 72 concrete nouns, and 72 adjectives that were used to modify the object of the sentence. Target words ranged in length from 3 to 11 letters, with a mean of 5.6. ${ }^{1}$ Two sentences were constructed for each target word: a coherent, semantically plausible sentence (though not highly constrained), and a second semantically incongruent sentence in which the semantic incongruity became apparent upon presentation of the target word (Table 1). All four types of semantically incongruous sentences were created by violating selectional restrictions at specific points along the sentence, but retaining grammaticality. Thus, the item creating the incongruity could have been one of the following: the first adjective (A1), the verb (V), the noun (object of the sentence) $(N)$ or the second adjective (A2). In the case of adjective incongruities, the transitive relations remained plausible, whereas in the verb and noun incongruities, the meaningful relation between the subject and the (direct or indirect) object was breached.

As mentioned in the introduction, Hebrew adjectives normally follow the noun they are modifying, so that in the case of the noun incongruity, the noun appears before the adjective in the sentence, and is, therefore, the word causing the incongruity. Similarly, in the A1 incongruity, a congruent subject noun precedes the adjective, so that the adjective is the word causing the incongruity.

Because the existent word-frequency counts in Hebrew are not sufficiently valid and comprehensive, we were unable to precisely match the lexical properties of the various stimulus categories. Therefore, while nevertheless trying to roughly match the frequency of words across categories, we decided to have the exact same target words appear in the congruent and incongruent conditions across participants, so that each word would, in effect, be its own control. Thus, in the present experiment, the target items remained constant and we manipulated the sentence frames

${ }^{1}$ In Hebrew vowels are mostly represented by diacritic marks. in which they were embedded to render them either plausible or incongruent.

The cloze probability of the target items in the coherent sentences was examined by a questionnaire administered to 48 undergraduates who participated neither in the plausibility judgment (see below) nor in the ERP study. Note that the procedure here differed from standard cloze probability ratings, because participants were asked to predict intermediate words rather than the final word of the sentence. Because we were interested in the degree to which target words could be predicted based upon the preceding context available to them (as would be the case in the actual experiment, when sentences are presented word by word) the questionnaires included only the fragment of the sentence leading up to the relevant position. The mean cloze probability of the target items in the coherent sentence frames was very low for all word types (below $5 \%$ ), and did not differ significantly by word type $(p>.1)$. Because target items were incongruent in the anomalous sentences, it is not surprising that the cloze probability in this condition always equaled zero (Table 2).

\section{Sentence Plausibility Rating}

The psycholinguistic impact of semantic violations of different types was examined by comparing ratings of sentence plausibility. This comparison was aimed at validating our hypothesis that the impact of a semantic incongruity would vary with the role of the incongruent word in the sentence. Because collecting ratings for each and every one of the 576 sentences used in this study was not feasible, we randomly sampled 24 sentences from each condition (coherent, and A1, Verb, Noun, and A 2 incongruities) for a total of 120 sentences. ${ }^{2}$ These sentences were randomized twice, and each version of the resulting questionnaire was presented to 16 participants, who were requested to rate how much the meaning of each sentence is plausible on a scale of 1 (makes no sense) to 7 (makes perfect sense). Participants were native Hebrew speakers who did not participate in the cloze probability rating or in the ERP study and received course credit or payment. As can be seen in Table 3, the results of the plausibility ratings confirmed our initial intuition regarding the differential degree of incongruity caused by the local and global semantic anomalies introduced in our materials.

Analysis of the ratings was performed using a one-way ANOVA over items, where the mean rating of the participants for each sentence in each condition was calculated. The plausibility ratings of sentences from the different conditions were found to differ significantly from each other, $F(4,115)=87.6$, $M S E=107.2, p<.001$. Planned contrasts demonstrated that coherent sentences were rated as being significantly more plausible than sentences sampled from all other conditions, $t(117)=16.1, p<.001$. Further, sentences with adjective incongruities were rated as being significantly more plausible than sentences with noun and verb incongruities, $t(117)=7.4$, $p<.001$, corroborating our initial hypothesis. Finally, the two adjective conditions did not differ significantly from each other, $t(46)=1.8, p>.7$, but verb incongruities were found to lead to greater implausibility than did noun incongruities, $t(46)=2.5$, $p<.05$ ), hinting to the crucial role of verbs in determining sentence meaning.

\footnotetext{
${ }^{2}$ The actual questionnaires included 120 additional sentences, of the same structure, which were normed for the purposes of a different project.
} 
Table 1. Examples of Coherent and Semantically Incongruent Sentences ${ }^{a}$

\begin{tabular}{|c|c|c|c|}
\hline Word class (target) & Sentence type & Sentence (English structure) & Sentence in Hebrew word order \\
\hline \multirow[t]{2}{*}{ Adjective 1 (Scholarly) } & Congruent & The scholarly professor lectured in the large hall & The professor scholarly lectured in the hall large \\
\hline & Incongruent & The scholarly bear slept in the warm cave & The bear scholarly slept in the cave warm \\
\hline \multirow[t]{2}{*}{ Verb (Fired) } & Congruent & The new manager fired the lazy worker & The manager new fired the worker lazy \\
\hline & Incongruent & The shiny scissors fired the burly coach & The scissors shiny fired the coach burly \\
\hline \multirow[t]{2}{*}{ Noun (Lock) } & Congruent & The skilled thief opened the heavy lock & The thief skilled opened the lock heavy \\
\hline & Incongruent & The excited woman thanked the old lock & The woman excited thanked the lock old \\
\hline \multirow[t]{2}{*}{ Adjective 2 (Prickly) } & Congruent & The happy child is rolling on the prickly grass & The child happy is rolling on the grass prickly \\
\hline & Incongruent & Tall Susan is eating a prickly tomato & Susan tall is eating a tomato prickly \\
\hline
\end{tabular}

${ }^{a}$ The word creating the incongruity is underlined.

\section{EEG Recording}

The EEG was recorded from 48 tin electrodes mounted on a custom made cap $(E C I)$ and amplified $\times 20,000$ with an analog band-pass filter of 0.1 to $70 \mathrm{~Hz}$ by SAI battery-operated amplifiers. The recording sites were based on the extended 10-20 system (Figure 1). EOG was recorded by two electrodes, one located over the outer canthus of the right eye and the other over the supraorbital region of the same eye. Both the EEG and the EOG were referenced to the tip of the nose.

The EEG was continuously sampled at $250 \mathrm{~Hz}$ and stored for off-line analysis. For ERP averaging, the EEG was parsed to 1000-ms epochs, starting $100 \mathrm{~ms}$ before the target stimulus. Epochs with EEG or EOG exceeding $\pm 100 \mu \mathrm{V}$ were excluded from averaging. The epochs were averaged separately for each stimulus condition, resulting in eight averaged ERPs per participant. All ERPs were based on a minimum of 24 trials per condition. The baseline was adjusted by subtracting the mean amplitude of the prestimulus period of each ERP from all the data points in the epoch. For statistical analyses, frequencies lower than $0.5 \mathrm{~Hz}$ and higher than $20 \mathrm{~Hz}(-3 \mathrm{~dB})$ were digitally filtered out after averaging.

\section{Procedure}

The experiment was conducted in an electrically shielded, dimly lit and sound-attenuated room. Electrode impedance was kept below $5 \mathrm{k} \omega$ throughout the experiment. Responses were given on a response box, using the index fingers of both hands. No special instructions were given regarding the structure of the sentences.

Sentences were presented word by word at the center of the screen, within a rectangular frame. Trials began with a fixation point at the center of the screen for $500 \mathrm{~ms}$, which was followed by a 100 -ms blank screen and then the first word of the sentence. Each word of the sentence was exposed for $750 \mathrm{~ms}$, with an interval of $100 \mathrm{~ms}$ between each two successive words. The sentence-final word appeared with a period and was followed by a 1.5-s blank screen, after which appeared either a probe question or the fixation point initiating the next trial. Probe questions appeared on the screen in full and never exceeded one line of text.

Table 2. Mean Cloze Probabilities (SEM) for Coherent and Anomalous Sentences

\begin{tabular}{lcc}
\hline \hline Sentence condition & Coherent & Anomalous \\
\hline Adjective1 & $2.3 \%(0.5)$ & 0 \\
Adjective2 & $4.1 \%(0.7)$ & 0 \\
Noun & $4.9 \%(0.8)$ & 0 \\
Verb & $3.4 \%(0.6)$ & 0 \\
\hline \hline
\end{tabular}

The questions remained on the screen until the participant responded. Participants received feedback for each answer: whether it was correct or incorrect and their overall percent of correct answers for the current block.

The session started with eight practice sentences, after which the 288 experimental sentences were presented. The sentences of each experimental list were randomized once, and presented in six blocks of 48 sentences each. Two filler sentences were added at the beginning of each experimental block. Breaks were introduced between the experimental blocks.

\section{Results}

Four participants were excluded from the analysis due to excessive eye movements or noisy data. The following results are, therefore, based on 16 participants, who had at least 24 uncontaminated trials in each experimental condition. Although several peaks and troughs are visible in each waveform, the most conspicuous difference between the ERPs elicited by semantically incongruent and congruent words was the N400 component, which was considerably more negative in response to incongruent words (Figure 2). In all ANOVAs the GreenhouseGeisser correction was applied whenever there was more than one degree of freedom. We report uncorrected degrees of freedom, $\varepsilon$ values, and adjusted $p$ values.

We set out to by examining the congruency and word type effects in the following three time epochs: $300-500 \mathrm{~ms}$ for the main N400 effects, $100-300 \mathrm{~ms}$ for earlier effects and 500-700 ms for late effects. For this analysis we calculated the mean amplitudes over the midline electrodes $(\mathrm{Fz}, \mathrm{FCz}, \mathrm{Cz}, \mathrm{CPz}, \mathrm{Pz}, \mathrm{POz})$, the sites at which the N400 effect was maximal. Thus, we submitted the mean amplitudes to a three-way within-participant analysis with the following factors: Epoch (early, middle, late), Congruity (coherent, anomalous), and Word Type (adjective 1, verb, noun, adjective 2). The two-way interaction between Epoch and Congruity was significant, $F(2,30)=15.1, p<.001, \varepsilon=.9$.

Table 3. Mean Plausibility Ratings (SEM) for Coherent and Four Types of Anomalous Sentences ${ }^{a}$

\begin{tabular}{lc}
\hline \hline Sentence condition & Rating \\
\hline Coherent & $6.68(.08)$ \\
Adjective1 & $3.47(.22)$ \\
Adjective2 & $4.03(.21)$ \\
Noun & $2.62(.26)$ \\
Verb & $1.86(.16)$ \\
\hline \hline
\end{tabular}

${ }^{\mathrm{a}}$ Ratings were between 1 (makes no sense) to 7 (perfectly coherent). 


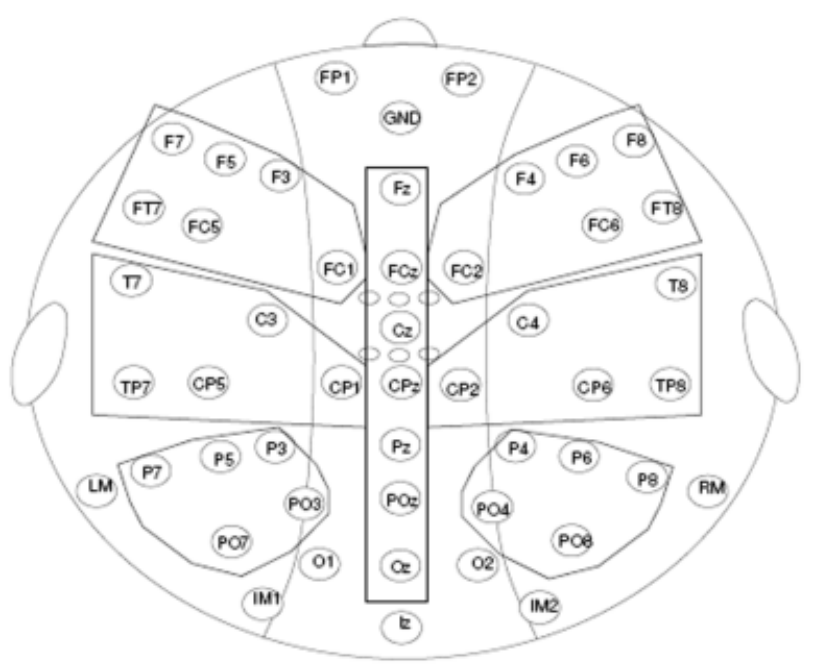

Figure 1. Electrode montage and ROIs.

Post hoc comparisons showed that the Congruity effect was not significant for the 100-300-ms epoch, $p>.05$, but it was significant for the 300-500-ms epoch, $F(1,15)=32.0, p<.001$, and smaller but still significant in the 500-700-ms epoch, $F(1,15)=22.0, p<.001$, in both cases due to more negative amplitudes for anomalous than for coherent items.

We interpret the congruity effect evident in the late window as a continuation of the N400 congruity effect originating in the 300-500-ms time window. Therefore, all further detailed analyses were carried out only on the $300-500-\mathrm{ms}$ epoch. This range encompasses the peak of the N400 at all sites, and conforms to the range of analysis reported in previous N400 studies (e.g., Holcomb, 1993).

The type of semantic incongruity modulated the N400 congruity effect in the 300-500-ms epoch at central and lateral sites.
As shown by Figure 3, the N400 effect (i.e., the difference between the ERP elicited by congruent and incongruent words), appeared larger when the semantically incongruent word was a noun or a verb than when it was an adjective. In fact, when the semantic incongruity was induced by the second adjective (the last word in the sentence, which modified the object) the N400 effect is not evident.

Peaking at midline sites, the distribution of the semantic congruence effect, at least for some anomaly types, was evident over the lateral cortex as well (Figure 4). Therefore, to reduce the degrees of freedom to a manageable number and yet analyze the entire distribution, the ERPs were collapsed into six regions of interest (ROIs): frontal-left (F7, F5, F3, FT7, FC5, FC1), frontal-right (F8, F6, F4, FT8, FC6, FC2), central-left (T7, C3, TP7, CP5, CP1), central-right (T8, C4, TP8, CP6, CP2), posterior-left (P7, P5, P3, PO7, PO3), and posterior-right (P8, P6, P4, PO8, PO4). These regions are labeled FL, FR, CL, CR, PL, and PR, accordingly. The analyses including ROI as a factor enabled comparison of distribution effects across hemispheres and the anterior/posterior axis. In addition, we analyzed the congruency effect along the midline, including in this analysis six sites $(\mathrm{Fz}$, $\mathrm{FCz}, \mathrm{Cz}, \mathrm{CPz}, \mathrm{Pz}, \mathrm{POz}$ ), as further exploration of the pattern observed at the sites at which the N400 effect was maximal.

ANOVA with repeated measures was used to investigate the congruency effect in each word type. For the midline, the ANOVA included three factors, Congruity (congruent, incongruent), Word Type (adjective 1, noun, verb, adjective 2), and Site. In a second ANOVA, we examined distributional factors. Hence, this ANOVA had four factors, Congruity (congruent, incongruent), Word Type (adjective 1, noun, verb, adjective 2), Hemisphere (left, right), and Sagittal Distribution (frontal, central, parietal).

As stated above, the ANOVA at midline showed that the mean amplitude of the N400 was significantly more negative in the incongruent $(-2.7 \mu \mathrm{V})$ than in the congruent condition $(0.1 \mu \mathrm{V}), F(1,15)=20.7, p<.001$. However, as revealed by significant interactions, this effect was modulated by Word Type

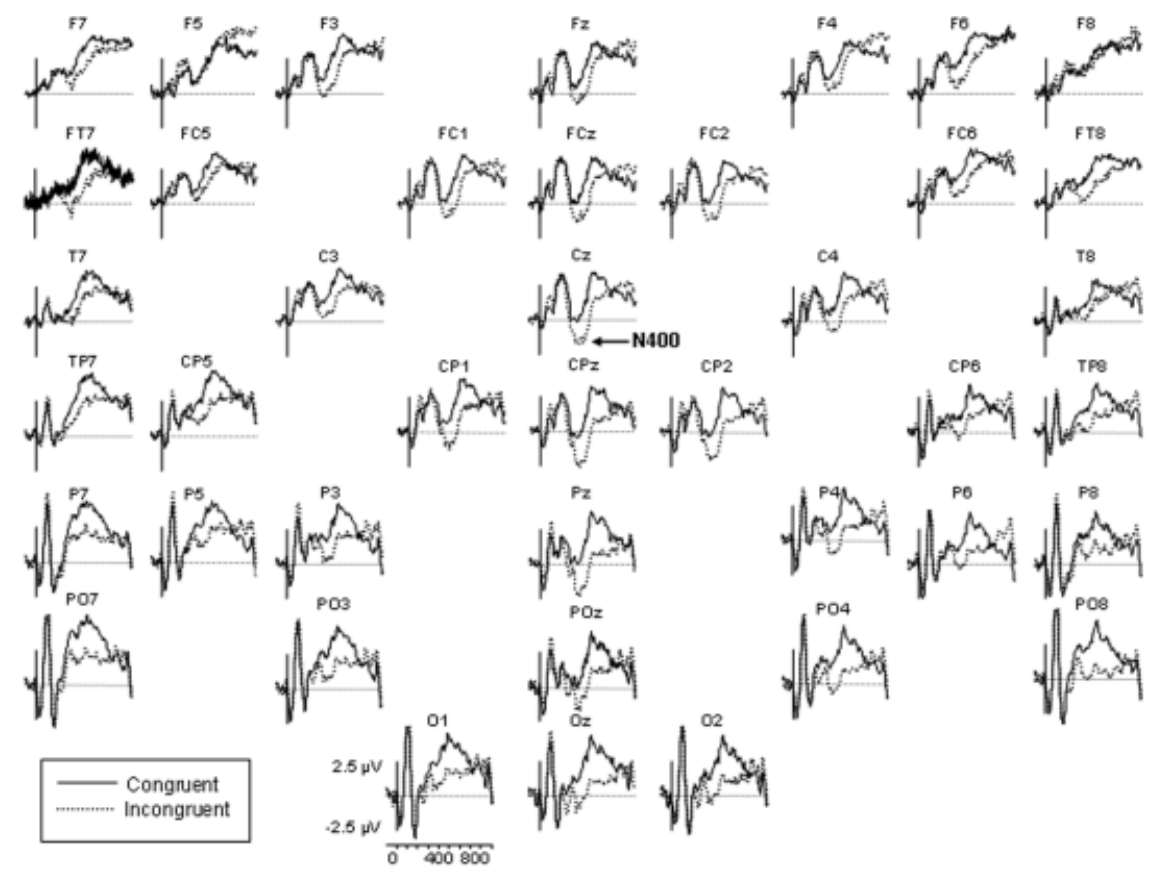

Figure 2. ERP waveforms elicited by semantically congruent and incongruent words. Data is presented prior to digital filtering. 

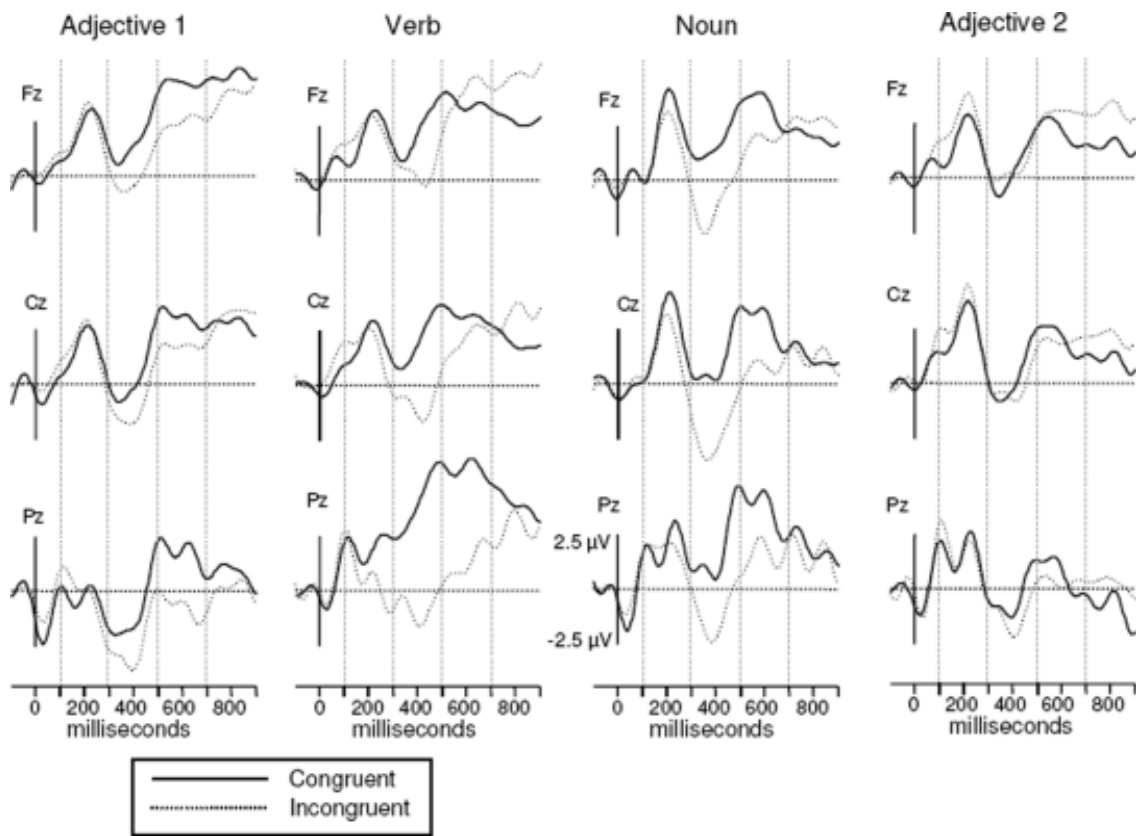

Figure 3. ERP waveforms elicited by congruent and incongruent words at midline, for each anomaly type. For easy visualization, data were low-pass filtered at $8 \mathrm{~Hz}$, reducing alpha band.

(see Figure 3), $F(3,45)=2.9, p<.05, \varepsilon=.8$. Post hoc exploration of the interaction revealed that the Congruity effect was significant for adjective 1, verb, and noun conditions, but not for the adjective 2 condition, $F(1,15)=6.9, p<.025, F(1,15)=17.6$, $p<.001, F(1,15)=14.2, p<.0025$, and $F(1,15)<1$, respectively. Furthermore, as revealed by a one-way ANOVA followed by planned contrasts, the Congruity effect was smaller for adjective 1 than for both the verb and noun conditions, which did not differ significantly from each other, $F(1,15)=5.1, p<.05$ and $F(1,15)<1$, respectively. Finally, the interaction between Word Type and Site was significant, $F(15,225)=12.0, p<.001, \varepsilon=.24$, due to the fact that whereas for nouns and adjectives in both sentence positions, amplitudes over frontal areas were generally less negative than over central and posterior areas, $p<.05$, for verbs the pattern was reversed, with greater negativities displayed over frontal regions, $p<.05$.

The pattern of the results revealed by the four-way analysis was very similar (see Table 4). The four-way ANOVA showed a significant Congruity $\times$ Word Type interaction, $F(3,45)=3.1$, $p<.05, \varepsilon=.9$. As in the analysis of midline sites, post hoc contrasts showed that the Congruity effect was significant for the adjective 1 , noun, and verb conditions, all $p<.01$, but not for the

\section{Mean voltage distribution, $300-500 \mathrm{msec}$ from stimulus onset}

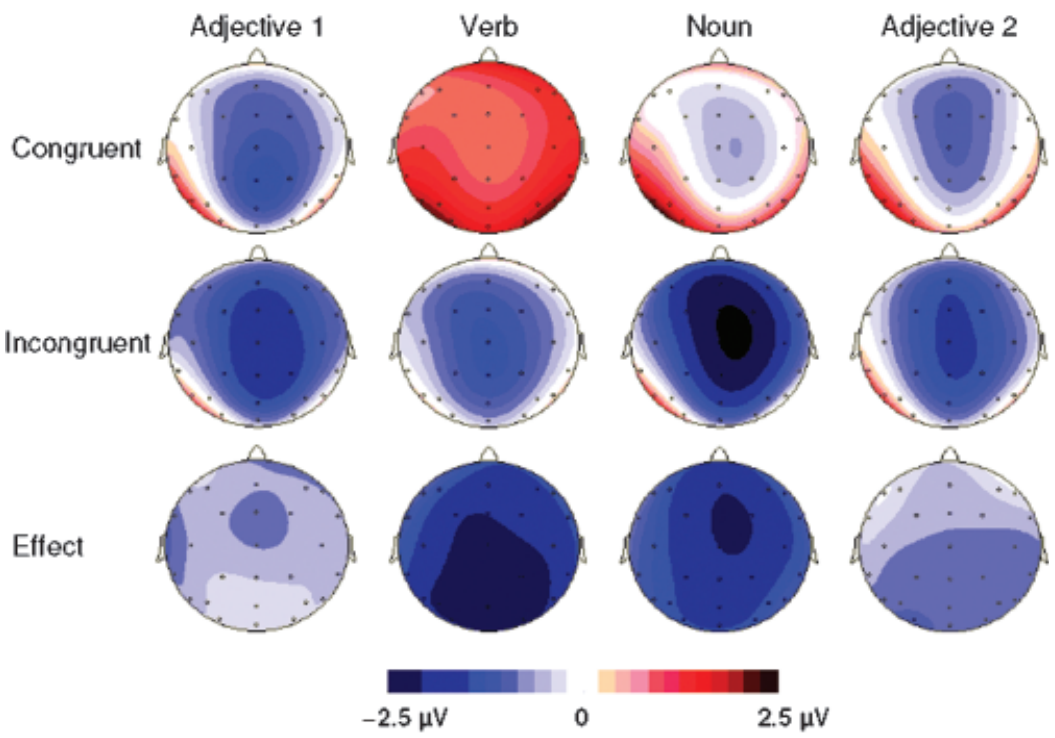

Figure 4. Mean voltage distributions at 300-500 ms for congruent and incongruent words by anomaly type and the congruency effect. 
Table 4. Mean Amplitudes (in Microvolts) Calculated for ROI in Each Experimental Condition

\begin{tabular}{|c|c|c|c|c|c|c|c|}
\hline \multirow[b]{2}{*}{ Anomaly } & & \multicolumn{3}{|c|}{ Left hemisphere } & \multicolumn{3}{|c|}{ Right hemisphere } \\
\hline & & FL & $\mathrm{CL}$ & PL & FR & CR & PR \\
\hline \multirow[t]{3}{*}{ Adjective 1} & Congruent & 1.00 & 1.87 & -0.15 & 0.87 & -0.78 & -1.01 \\
\hline & Incongruent & -0.89 & -1.69 & -2.11 & -0.94 & -2.45 & -3.37 \\
\hline & N400 effect & 1.89 & 3.56 & 1.96 & 1.81 & 1.67 & 2.36 \\
\hline \multirow[t]{3}{*}{ Verb } & Congruent & -0.46 & 1.87 & 3.80 & -0.72 & 0.86 & 2.22 \\
\hline & Incongruent & -3.18 & -1.69 & -0.89 & -3.35 & -2.66 & -2.34 \\
\hline & N400 effect & 2.72 & 3.56 & 4.69 & 2.63 & 3.52 & 4.56 \\
\hline \multirow[t]{3}{*}{ Noun } & Congruent & 1.87 & 1.62 & 1.61 & 1.47 & 0.45 & 0.39 \\
\hline & Incongruent & -0.21 & -0.46 & 0.01 & -1.07 & -1.61 & -1.54 \\
\hline & N400 effect & 2.08 & 2.08 & 1.60 & 2.54 & 2.06 & 1.93 \\
\hline \multirow[t]{3}{*}{ Adjective } & Congruent & 1.23 & 0.64 & -0.11 & 0.52 & -0.40 & -0.88 \\
\hline & Incongruent & 1.17 & 0.46 & -0.36 & 0.89 & -0.86 & -1.56 \\
\hline & N400 effect & 0.06 & 0.18 & 0.25 & -0.37 & 0.46 & 0.68 \\
\hline
\end{tabular}

adjective 2 condition, $F<1$. In addition, there was a significant interaction between the Word Type and Sagittal distribution effects, $F(6,90)=19.5, p<.001, \varepsilon=.433$.

The interaction between Word Type and Sagittal distribution was further examined by post hoc two-way ANOVAs, performed separately for each Word Type condition. The factors in these ANOVAs were Hemisphere and Sagittal Distribution. These analyses were then followed by planned contrasts, comparing the three levels of Sagittal Distribution.

The main effect of Sagittal Distribution was significant for the adjective 1 and adjective 2 Word Type conditions, $F(2,30)=5.8$, $p<.01, \varepsilon=.55, F(2,30)=9.6, p<.01, \varepsilon=.56$, respectively, but not for the noun and verb conditions, $p>1$. Post hoc contrasts revealed that the ERPs elicited by adjectives followed the same pattern evident at the midline sites - amplitudes were more negative at posterior than at anterior sites, $F(1,15)=8.1, p<.02$ and $F(1,15)=10.9, p<.005$, for adjective 1 and adjective 2 , respectively. Finally, there was a significant interaction between Hemisphere and Sagittal Distribution for the verb condition, $F(2,30)=7.3, p<.01, \varepsilon=.65$, due to the fact that at anterior sites the left hemisphere showed greater negativity than the right $(-1.8$ vs. $0.19 \mu \mathrm{V}$ for left and right, respectively) whereas at posterior sites the pattern was reversed $(0.8 \mathrm{vs}$. $-0.57 \mu \mathrm{V}$ for left and right, respectively).

\section{Latency Analysis}

We compared the latency of the peak of the N400 component across the four incongruous conditions. We identified the latency of the most negative point in the waveform between 250 and $600 \mathrm{~ms}$ over midline sites per participant per condition. The mean latency across conditions was $400 \mathrm{~ms}$ with the verb and noun conditions having longer latencies (409 and $424 \mathrm{~ms}$, respectively) than the adjective conditions (377 and $392 \mathrm{~ms}$ for A1 and A2, respectively). We then performed one-way repeated measures ANOVA on these data, with four levels: incongruent adjective1, verb, noun, and adjective2. This analysis showed a trend toward a main effect of incongruity type, but did not reach statistical significance, $F(3,45)=2.3, p=.08$. We therefore did not analyze the latency data any further.

\section{Discussion}

The current study examined the relative impact of nouns, verbs, and adjectives in shaping sentence meaning as revealed by sen- tence plausibility ratings and by the magnitude of the N400 effect elicited online during sentence integration processes. Plausibility ratings demonstrated that sentences including a semantically incongruent adjective were judged to be more plausible than sentences including a semantically incongruent noun or verb. Thus, the important role of verbs in sentence comprehension (Dowty, 1991; Ferretti et al., 2001) was validated once again: The verb incongruities reduced sentence plausibility to the greatest extent, compared with incongruities occurring in other parts of speech.

In a similar vein, the N400 congruity effect was significantly larger for semantically incongruent nouns and verbs than for incongruent adjectives. In fact, when the semantically incongruent adjective was the last word in the sentence, modifying the object of the sentence, there was no significant N400 effect at all.

The presently observed differences in the N400 effect associated with words of different class is in accord with our hypothesis that the incongruity types examined in our study vary in the degree to which they disrupt the process of sentence integration. To reiterate the argument presented in the introduction, incongruent adjectives created only a local interruption in the processing of sentence meaning, whereas incongruent nouns and verbs disrupted the entire argument structure of the sentence, and therefore posed a greater integrative challenge, leading to larger N400 effects. Phrased differently, during the analysis of incoming information, certain thematic role slots are awaiting their completion (Carlson \& Tanenhaus, 1988; Ferretti et al., 2001; Frazier \& Clifton, 1996; Frazier \& Rayner, 1982). In the case of the simple transitive sentences used in the present study, once the subject of the sentence is specified, the slot for a predicate becomes available. Further, once a transitive verb is introduced as predicate, a slot opens for the object of the sentence (for recent reviews, see Carpenter et al., 1995; Clifton \& Duffy, 2001). Thus, when the locus of the incongruity is at the verb or noun, the violation of the selectional restrictions leads to a significant mismatch - the incoming information cannot be accommodated to fill the pending thematic role.

In contrast to the above, when an incongruent adjective is encountered, the basic argument structure of the sentence is still tenable. In a sense, the problematic adjective may be overlooked, so to speak, without causing the entire integrative process to fail. Our finding that an incongruity at the point of the early adjective, modifying the subject, is more detrimental than an incongruity at the late adjective, modifying the object, fits well with this explanation. Once the subject of the sentence has been introduced, there are thematic roles awaiting assignment. When the adjective is encountered, its appropriateness for the unassigned roles must be determined. When the grammatical class of the adjective is computed, the predicate slot remains unfulfilled. So despite the local mismatch between the subject and the modifying adjective, there still remains an opportunity for salvaging the general structure of the sentence. And indeed, the subsequent verb and noun allow the completion of a basic argument structure.

The case of the sentence final adjective violation, for the object-modifying adjective, is even more illuminating. At this point in the process, the crucial thematic roles have been successfully assigned. Our results demonstrate that because there are no more unassigned thematic roles at this point, the online integration process is less sensitive to this type of late incongruity. It almost seems as though the incongruent information is effectively "discarded," and the semantic system does not engage in any significant attempt to successfully integrate it with the preceding context. 
Further evidence regarding the local and limited scope of adjectives in sentence integration comes from a comparison of the N400 peaks to congruent adjectives presented early in the sentence and to adjectives presented at the sentence-final position (Figure 5). Notably, the present data show no significant difference between these conditions, $p>.7$, in contrast with previous demonstrations of a decline in the amplitude of the N400 component elicited by contextually congruent open class as a function of word position in the sentence (e.g., Kutas, Van Petten, \& Besson, 1988). These effects of ordinal position are thought to reflect the accumulation of semantic context that facilitates integration: The amplitude of the N400 indexes the effort required to integrate every successive word with the representation constructed. Congruent words that come late in the sentence benefit from the previous semantic context, whereas earlier words have little context and thus elicit larger N400 s (Van Petten \& Kutas, 1991). However, previous work in this domain examined mostly sentence-final nouns, which are obligatory for the completion of the sentence structure and thematic role assignment. It might be the case, then, that the general accretion of semantic context is more significant for mandatory sentence constituents that participate in determining the global semantic structure of the sentence than for optional sentence constituents, which play a more limited local semantic role.
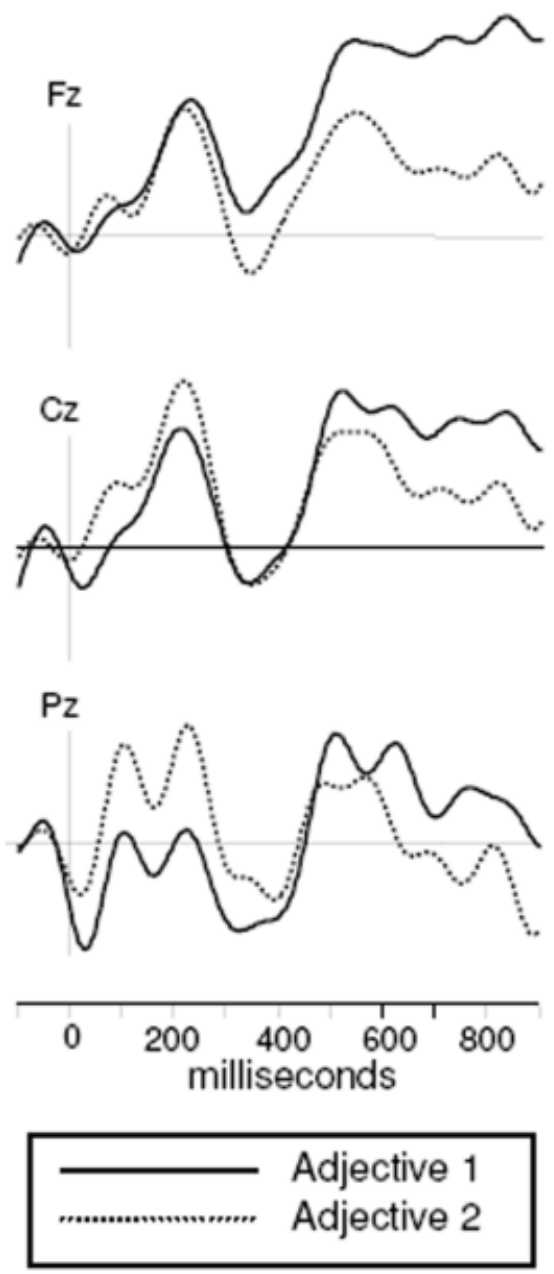

Figure 5. ERP waveforms at midline elicited by congruent adjectives in the early and sentence-final positions. For easy visualization, data were low-pass filtered at $8 \mathrm{~Hz}$, reducing alpha band.
Along the same lines, previous work has found that semantic incongruities occurring at nouns appearing in later stages of sentence processing lead to larger N400 effects than early incongruities. Conversely, the present study examined sentence-final adjectives, which are optional components as far as sentence structure is concerned, and found no N400 congruity effects at the sentence-final position. This finding, once again, illustrates the differential effect of incongruent words of various types on sentence integration.

One should note, however, that the N400 patterns do not perfectly match the results obtained from the plausibility rating task. The major finding of greater meaning disruption introduced by mandatory as opposed to optional incongruities is present in both methods, but there are variations. Specifically, sentences including an incongruent second adjective were rated as being as implausible as those including an incongruent first adjective, attesting to participants' ability to detect these incongruities. Conversely, the adjective 2 incongruous sentences did not lead to a significant N400 effect. Similarly, anomalous verbs were rated as creating less plausible sentences than anomalous nouns, whereas the magnitude of the N400 effect for these two word types was comparable. We propose that these differences might reflect the fact that plausibility rating is an off-line judgment, and might rely on processes that go beyond the initial extraction of sentence meaning, which is reflected in the online ERP measures. A study using timed or speeded plausibility ratings might shed light on the early and late processes contributing to the final judgment and the degree to which they are shared or different from the mechanism giving rise to the $\mathrm{N} 400 .^{3}$

Previous work has shown that the relation between an incongruent item and an expected item can modulate the N400 effect to the incongruity (Kutas \& Hillyard, 1980). However, there has not been much focus on other factors that might influence the degree of incongruity caused by different items. In a study that addressed similar issues, Federmeier and colleagues (2000) reported that an incongruity at the point of an expected verb evoked a larger N400 than an incongruity at the point of an expected noun. However Federmeier et al. did not use semantically incongruent items of the same word type, and thus although the present results are commensurate with their findings, the studies are not directly comparable. Furthermore, that study did not examine the response to semantically incongruent adjectives, and thus did not compare the effects of incongruities in mandatory and optional sentence constituents.

We interpret the sensitivity of the N400 effect to the syntactic/ thematic properties of incongruent words used in this study as reflecting the different roles that they normally play in computing sentence meaning and the integrative burden posed by such incongruities, rather than as a sensitivity of the N400 to syntactic word type distinctions per se (and see Friederici, Gunter, Hahne, $\&$ Mauth, 2004). This interpretation receives support from the difference we found in the N400 effect for the two adjective incongruities introduced, and specifically the absence of an N400 effect for the incongruent sentence-final adjective. We take this as evidence that it is the role of the word in ongoing sentence comprehension, and not its syntactic class, that determines the degree of interruption caused by an incongruity.

${ }^{3}$ It should be noted that plausibility ratings were collected for only a sample of the sentences presented in the ERP study. Though the sentences were randomly selected, it is still possible that the difference in plausibility patterns is a result of the specific sample selected. 
Finally, as a side issue, it is worth noting that we found evidence for differences in the distribution of the N400 for the word types examined. This finding can be attributed mainly to the divergence of the N400 distribution for verbs from the N400 distribution for nouns and adjectives. Recent PET studies have reported mixed results regarding differences in the brain activity in response to nouns and verbs (Black \& Chiat, 2003; Cappa \& Perani, 2003). Some studies have found verb-specific activation patterns, especially in the dorsolateral frontal and lateral temporal cortex of the left hemisphere (Perani et al., 1999), whereas others report a large degree of overlap in the areas processing nouns and verbs (e.g., Tyler, Russell, Fadili, \& Moss, 2001; for a recent review, see Shapiro \& Caramazza, 2003). Electrophysiological evidence has also been inconclusive. Pulvermuller and colleagues (1999) reported differences in brain responses to isolated nouns and verbs, with nouns eliciting posterior activity and verbs generating more central activity. However, they claim that these results might reflect the typical semantic associations of nouns and verbs and not word class per se. A recent study of nouns and verbs processed in sentential context (Federmeier et al., 2000) found greater N400 negativity over central areas for nouns when compared to verbs. Adjectives were not investigated in any of the above studies.

The present results are in partial accord with findings showing more anterior activity for verbs, and also some evidence agreeing with the previous findings of predominant left-hemisphere negativity for verbs. The pattern of activity that we found for adjec- tives, namely more posterior negativities, has not been previously reported. However, the present experiment was not designed to investigate the effect of word class per se, and the materials we used were not sufficiently controlled to allow the desired degree of confidence that the observed differences are indeed the consequence of word class. Thus, the frequency, length, and concreteness of words from the various word classes were not perfectly matched. For example, to the extent that adjectives tend to be less concrete than nouns, the finding of greater posterior negativities for this word class may be attributed to this factor, specifically in the incongruent sentence conditions, in accordance with previous findings (Holcomb, Kounious, Anderson, \& West, 1999). We therefore leave this issue for future investigation.

In conclusion, the present study confirmed that nouns, verbs, and adjectives play different roles in the computation of sentence meaning and showed that the online semantic integration process is sensitive to this difference. All incongruities are therefore not created equal: Incongruities introduced in optional sentence constituents, such as adjectives in this case, create less of an integrative burden than incongruities in mandatory sentence constituents, such as nouns and verbs. This pattern was evident both in plausibility ratings and in the modulation of the N400 component. We interpret these findings as suggesting that online sentence integration processes are sensitive to the distinct roles played by words of different types in shaping sentence meaning, and not as a demonstration of N400 sensitivity to syntactic word class per se.

\section{REFERENCES}

Altmann, G. T. M., \& Kamide, Y. (1999). Incremental interpretation at verbs: Restricting the domain of subsequent reference. Cognition, 73, 247-264.

Bentin, S., Mouchetant-Rostaing, Y., Giard, M. H., Echallier, J. F., \& Pernier, J. (1999). ERP manifestations of processing printed words at different psycholinguistic levels: Time course and scalp distribution. Journal of Cognitive Neuroscience, 11, 235-260.

Black, M., \& Chiat, S. (2003). Noun-verb dissociations: A multi-faceted phenomenon. Journal of Neurolinguistics, 16, 231-250.

Brown, C., Hagoort, P., \& ter Keurs, M. (1999). Electrophysiological signatures of visual lexical processing: Open- and closed-class words. Journal of Cognitive Neuroscience, 11, 261-281.

Brown, M. C., \& Hagoort, P. (1999). On the electrophysiology of language comprehension: Implications for the human language system. In M. Crocker, M. Pickering, \& C. Clifton (Eds.), Architectures and mechanisms for language processing (pp. 213-237). Cambridge: Cambridge University Press.

Cappa, S. F., \& Perani, D. (2003). The neural correlates of noun and verb processing. Journal of Neurolinguistics, 16, 183-189.

Carlson, G. N., \& Tananhaus, M. K. (1988). Thematic roles and language comprehension. In W. Wilkins (Ed.), Thematic relations (pp. 263-288). New York: Academic Press.

Carpenter, P. A., Miyake, A., \& Just, M. A. (1995). Language comprehension: Sentence and discourse processing. Annual Review of Psychology, 46, 91-120.

Chomsky, N. (1981). Lectures on government and binding. Dordrecht: Foris.

Clifton, C., \& Duffy, S. A. (2001). Sentence and test comprehension: Roles of linguistic structure. Annual Review of Psychology, 52, 167-196.

DeLong, K. A., Urbach, T. P., \& Kutas, M. (2005). Probabilistic word pre-activation during language comprehension inferred from electrical brain activity. Nature Neuroscience, 8, 11171121.

Dowty, D. R. (1991). Thematic proto-roles and argument selection. Language, 67, 547-619.
Federmeier, K. D., Segal, J. B., Lombrozo, T., \& Kutas, M. (2000). Brain responses to nouns, verbs and class-ambiguous words in context. Brain, 123, 2552-2566.

Ferretti, T. R., McRae, K., \& Hatherell, A. (2001). Integrating verbs, situation schemas, and thematic role concepts. Journal of Memory and Language, 44, 516-547.

Fisher, C., Gleitman, H., \& Gleitman, L. R. (1991). On the semantic content of subcategorization frames. Cognitive Psychology, 23, 331-392.

Frazier, L., \& Clifton, C. (1996). Construal. Cambridge, MA: MIT Press.

Frazier, L., \& Rayner, K. (1982). Making and correcting errors during sentence comprehension: Eye movements in the analysis of structurally ambiguous sentences. Cognitive Psychology, 14, 178-210.

Friederici, A. D. (2004). The neural basis of syntactic processes. In M. S. Gazzaniga (Ed.), The cognitive neurosciences (3rd ed, pp. 789-801). Cambridge, MA: MIT Press.

Friederici, A. D., Gunter, T. C., Hahne, A., \& Mauth, K. (2004). The relative timing of syntactic and semantic processes in sentence comprehension. NeuroReport, 15, 165-169.

Frisch, S., Hahne, A., \& Friederici, A. D. (2004). Word category and verb-argument structure information in the dynamics of parsing. Cognition, 91, 191-219.

Grodner, D., Gibson, E., \& Tunstall, S. (2002). Syntactic complexity in ambiguity resolution. Journal of Memory \& Language, 46, $267-295$.

Hagoort, P. (2003). Interplay between syntax and semantics during sentence comprehension: ERP effects of combining syntactic and semantic violations. Journal of Cognitive Neuroscience, 15, 883-899.

Hinojosa, J. A., Martin-Loeches, M., \& Rubia, F. J. (2001). Eventrelated potentials and semantics: An overview and an integrative proposal. Brain and Language, 78, 128-139.

Holcomb, P. J. (1993). Semantic priming and stimulus degradation: An event-related brain potential analysis of semantic priming. Psychophysiology, 30, 47-62.

Holcomb, P. J., Kounious, J., Anderson, J. E., \& West, W. C. (1999). Dual-coding, context availability, and concreteness effects in sentence 
comprehension: An electrophysiological investigation. Journal of Experimental Psychology: Learning, Memory and Cognition, 25, 721-742.

Kaan, E., \& Swaab, T. Y. (2003). Electrophysiological evidence for serial sentence processing: A comparison between non-preferred and ungrammatical continuations. Cognitive Brain Research, 17, 621-635.

Kuperberg, G. R., Sitnikova, T., Caplan, D., \& Holcomb, P. J. (2003). Electrophysiological distinctions in processing conceptual relationships within simple sentences. Cognitive Brain Research, 17, 117-129.

Kutas, M., Federmeier, K. D., Coulson, S., King, J. W., \& Munte, T. F. (2000). Language. In J. T. Cacioppo \& L. G. Tassinary (Eds.), Handbook of psychophysiology (2nd ed, pp. 576-601). New York: Cambridge University Press.

Kutas, M., \& Hillyard, S. (1980). Event-related brain potentials to semantically inappropriate and surprisingly large words. Biological Psychology, 11, 99-116.

Kutas, M., \& Hillyard, S. (1984). Brain potentials during reading reflect word expectancy and semantic association. Nature, 307, $161-163$.

Kutas, M., \& King, J. W. (1996). The potentials for basic sentence processing: Differentiating integrative processes. In T. Inui \& J. L. McClelland (Eds.), Attention and performance 16: Information integration in perception and communication (pp. 501-546). Cambridge, MA: MIT Press.

Kutas, M., Lindamood, T., \& Hillyard, S. A. (1984). Word expectancy and event-related brain potentials during sentence processing. In $\mathrm{S}$. Kornblum \& J. Renquin (Eds.), Preparatory states and processes (pp. 217-237). Hillsdale, NJ: Erlbaum.

Kutas, M., \& Van Petten, C. K. (1994). Psycholinguistics electrified: Event-related brain potential investigations. In Handbook of psycholinguistics (pp. 83-143). New York: Academic Press.

Kutas, M., Van Petten, C. K., \& Besson, M. (1988). Event-related potentials asymmetries during the reading of sentences. Electroencephalography and Clinical Neurophysiology, 69, 218-233.

Levin, B. (1993). English verb classes and alternations: A preliminary investigation. Chicago: University of Chicago Press.

MacDonald, M. C., Pearlmutter, N. J., \& Seidenberg, M. S. (1994). The lexical nature of syntactic ambiguity resolution. Psychological Review, 101, 676-703.

McCarthy, G., Nobre, A. C., Bentin, S., \& Spencer, D. D. (1995). Language-related field potentials in the anterior-medial temporal lobe. Journal of Neurophysiology, 15, 1080-1089.

Munte, T. F., Wieringa, B. M., Weyerts, H., Szentkuti, A., Matzke, M., \& Johannes, S. (2001). Differences in brain potentials to open and closed class words: Class and frequency effects. Neuropsychologia, 39, 91-102.
Myers, E. B., \& Blumstein, S. E. (2005). Selectional restriction and semantic priming effects in normals and Broca's aphasics. Journal of Neurolinguistics, 18, 277-296.

Neville, H. J., Mills, D. L., \& Lawson, D. S. (1992). Fractionating language: Different neural subsystems with different sensitive periods. Cerebral Cortex, 2, 244-258.

Osterhout, L., Allen, M., \& McLaughlin, J. (2002). Words in the brain: Lexical determinants of word-induced brain activity. Journal of $\mathrm{Ne}$ urolinguistics, 15, 171-187.

Osterhout, L., \& Holcomb, P. J. (1995). Event-related potentials and language comprehension. In M. D. Rugg \& M. G. H. Coles (Eds.), Electrophysiology of mind (pp. 171-215). Oxford: Oxford University Press.

Perani, D., Cappa, S. F., Schnur, T., Tettamanti, M., Collina, S., Rosa, M. M., et al. (1999). The neural correlates of verb and noun processing: A PET study. Brain, 122, 2337-2344.

Pulvermuller, T., Lutzenberger, W., \& Preissl, H. (1999). Nouns and verbs in the intact brain: Evidence from event-related potentials and high-frequency cortical responses. Cerebral Cortex, 9, 497-506.

Shapiro, K., \& Caramazza, A. (2003). The representation of grammatical categories in the brain. Trends in Cognitive Science, 7, 201-206.

Titone, D. A., \& Salisbury, D. F. (2004). Contextual modulation of N400 amplitude to lexically ambiguous words. Brain \& Cognition, 55, 470-478.

Townsend, D. J., \& Bever, T. C. (2001). Sentence comprehension: The integration of habits and rules. Cambridge, MA: MIT Press.

Trueswell, J. C., Tanenhaus, M. K., \& Garnsey, S. M. (1994). Semantic influences on parsing: Use of thematic role information in syntactic ambiguity resolution. Journal of Memory \& Language, 33, 285-318.

Tyler, L. K., Russell, R., Fadili, J., \& Moss, H. E. (2001). The neural representation of nouns and verbs: PET studies. Brain, 124, 1619-1634.

van Berkum, J. J. A., Hagoort, P., \& Brown, C. M. (1999). Semantic integration in sentences and discourse: Evidence from the N400. Journal of Cognitive Neuroscience, 11, 657-671.

van Berkum, J. J. A., Zwitserlood, P., Hagoort, P., \& Brown, C. M. (2003). When and how do listeners relate a sentence to the wider discourse? Evidence from the N400 effect. Cognitive Brain Research, 17, 701-718

Van Petten, C. K., \& Kutas, M. (1991). Influences of semantic and syntactic context in open- and closed-class words. Memory \& Cognition, $19,95-112$

Warren, T., \& Gibson, E. (2002). The influence of referential processing on sentence complexity. Cognition, 85, 79-112.

(Received November 14, 2005; ACCePted May 15, 2006) 